different because of differences in epigenetic modification resulting from genomic imprinting. Certainly, we must look for a mechanism that either produces differences in mutation between parental alleles, or that selects for differences between them. The best molecular evidence for an imprinting process comes from experiments with transgenic mice, where several groups have reported examples of differences of DNA methylation between parental alleles" ${ }^{11}$. How there could be selective somatic mutation on the paternal allele of the RB gene is not clear from these studies. If the paternal allele is more methylated in most somatic cells, the increased frequency of mutation by deamination of 5-methylcytosine could contribute to selective mutation of the paternal gene. Both the methylation status of the paternal RB allele and the frequency of mutation at $\mathrm{CpG}$ dinucleotides can now be analysed with the RB gene in hand.

On the basis of the observations in Wilms tumour, Wilkins ${ }^{12}$ proposed that a transforming gene (TR) linked to the recessive Wilms gene on chromosome 11 is imprinted. Inactivation of the Wilms gene product derepresses this transforming gene and results in development of the tumour. However, the maternal allele of TR is rendered inactive by methylation imprinting and so it is only the combination of the inactive Wilms gene and the active (paternal) TR on the paternal chromosome that results in a tumour. Two assumptions are implicit in this model, and they should become testable. First, inactivation by imprinting of the maternal TR allele should be observed in most, if not all, cells of the embryonic kidney. Second, whenever the first mutation is in the maternal Wilms gene, a tumour should arise when somatic recombination between the Wilms locus and TR produces a hybrid chromosome with the Wilms mutation and the active (paternal) transforming gene. If this is correct, the information on location of the recombination breakpoints could also be used to map the position of the proposed TR gene.

Finally, we wish to draw attention to an alternative explanation based on a suggestion originally made by Sapienza et al. ${ }^{13}$. Based on various experimental observations, these authors proposed that imprinting by methylation is not retained in most cells in an embryo. In this explanation, tissues in the embryo are composed of many cells in which both alleles are active, and of rather a few cells that retain methylation differences between specific parental alleles. If we extrapolate from the findings with transgenes in the mouse, then in these cells the maternal allele is likely to be relatively inactive because of hypermethylation (see figure). If the first mutation in the Wilms gene or the RB gene occurs on the paternal copy in these cells, and is segregated into the correct target tissue, a focus of tumour cells will appear in kidney or bone. This focus greatly expands the number of target mitoses for the second event, through which the remaining allele is lost. The remaining (maternal) allele is not necessarily completely repressed by methylation; indeed varying degrees of reduction in activity can be envisaged that may even change during the growth of the focus. So, what remains in this model is the possibility that the 'second hit' does not happen. Perhaps this could explain a frequent observation in tumour biology, one also true for retinoblastoma and Wilms tumour - the finding of tumour-like lesions or foci in these tissues that have regressed (usually a chance discovery on autopsy).

Much of the present uncertainty about the true mechanism underlying the unequal representation of parental chromosomes in these tumours should soon be removed by the techniques of molecular biology. At least now that the RB gene has been cloned, various questions raised by the observations we have described here can now be addressed by looking closely at mutations, at transcription, and at methylation. The most exciting prospect is that the same principle may operate in many, if not all, recessive tumour syndromes. Further analysis of this principle may reveal more about the significance of genomic imprinting in the control of normal and malignant cell differentation.

Wolf Reik and M. Azim Surani are in the Department of Molecular Embryology, AFRC Institute of Animal Physiology and Genetics Research, Babraham, Cambridge CB2 4AT, UK.

1. Solter, D. A. Rev. Genet. 22, 127-146 (1988).

2. Cattanach, B. M. \& Kirk, M. Nature 315, $496(1985)$

3. Toguchida, J. et al. Nature 338, 156-158 (1989).

4. Schroeder, W.T. et al. Am. J. hum. Genet. 40, 413 (1987)

5. Mannens, M. et al. Hum Genet. $\mathbf{8 1}, 41-48$ (1988)

6. Ponder, B. Nature 335, 400-402 (1988).

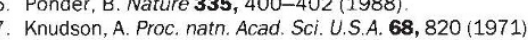

8. Knudson, A.G. \& Strong, L.C. J. natn. Cancer Inst. 48 313-324 (1972).

9. Comings, D.E. Proc. natn. Acad. Sci. U.S.A. 70, 3324 $3328(1973)$

10. Magenis. R.E. Am J. hum. Genet. 42, 529-533 (1988).

11. Surani, M.A.H et al Trends Genet 4, 59-62 (1988).

12. Wilkins, R.F. Lancet i, 329-331 (1988).

13. Sapienza, C. et al. Prog. Nucleic Acids Res. molec. Biol. (in the press).

\title{
An anniversary for atmospheric ozone
}

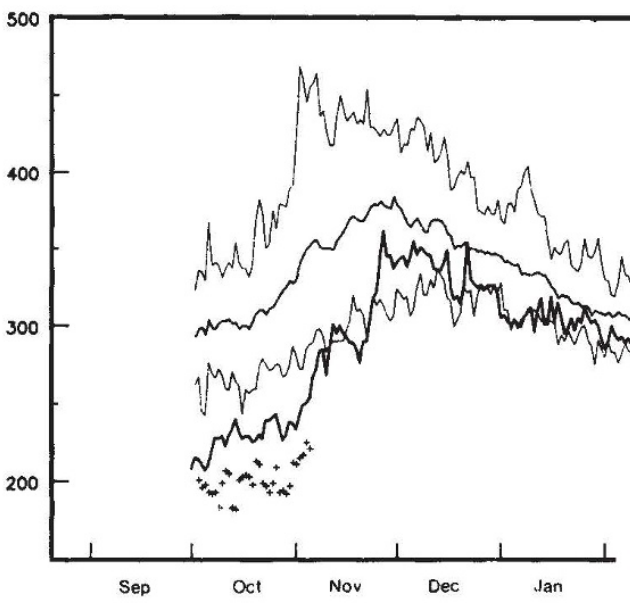

G. M. B. Dobson, who pioneered atmospheric ozone measurements, was born on 25 February 1889. The photograph was taken while Dobson was a member of the Meteorological Office Research Committee in 1955. His interest in ozone was aroused by his studies of meteor trails in the atmosphere, which led to his discovery of a high-temperature region at a height of about 50 kilometres. His realization that the source of energy for this warm layer is the absorption of ultraviolet radiation by ozone inspired him to spend the rest of his life studying atmospheric ozone. Dobson developed a technique for measuring ozone in which a photoelectric spectrophotometer is used to compare the absorption in the solar spectrum resulting from ozone at two ultraviolet wavelengths in the absorption band of ozone by allowing the radiation of each wavelength to strike the photocell. In the 1930s Dobson set up a network of ozonemeasuring stations throughout the world, many of which are still in existence. Indeed, the Antarctic ozone hole was discovered by a team from the British Antarctic Survey working at Halley Bay in Antarctica (Nature 315, 207; 1985; see graph) using a Dobson instrument to measure ozone. Satellite measurements of ozone began only in the late 1970s and so long-term changes and trends in atmospheric ozone concentration can be assessed only using the older, ground-based data. The satellite data, while providing more comprehensive global coverage, are prone to suffer long-term drifts in instrument calibration when in orbit. To compensate for this effect, therefore, the satellite data are normalized against the Dobson data.
Philippa Lloyd 